\title{
The Role of Early Mobilization in the Intensive Care
}

\section{Ana Zão*}

\author{
Physical and Rehabilitation Department, Centro Hospitalar do Porto, Porto, Portugal
}

*Corresponding author: Ana Zão, Physical and Rehabilitation Department, Centro Hospitalar do Porto, Largo do Prof. Abel Salazar, 4099-001 Porto, Portugal, E-mail: anazaomfr@gmail.com

\begin{abstract}
Background: The hospitalization in an Intensive Care Unit may lead to important structural and functional deficits, resulting in high morbidity and mortality. This functional decline is largely due to immobility.

Methods: Narrative review of the literature published until February 2018 in the Medline, Embase, Cochrane Library, Web of Science and Scopus databases.

Results: Amyotrophy, muscle strength deficit, sarcopenia, pain, contractures and osteoporosis are some of the musculoskeletal complications frequently observed in critically ill patients. However, the negative effects of immobility are rarely limited to a single system. Numerous other comorbidities may result from hospitalization in an Intensive Care Unit: Cardiovascular, respiratory, genito-urinary, gastro-intestinal, endocrine and metabolic, immune, cutaneous, neurological and cognitive-behavioral. The etiology is multifactorial and several risk factors have been described. The evidence about the benefits of early mobilization and exercise in critically ill patients is growing in the literature, focusing on different systems.

Conclusion: Immobility syndrome, myopathy and neuropathy are serious complications commonly found in the Intensive Care Unit, which delay the recovery of the underlying disease itself, can lead to significant functional limitations, restriction of activities, reduction of participation and integration of patients in their social, professional and family contexts.
\end{abstract}

\section{Keywords}

Mobilization, Intensive care, Exercise, Myopathy, Neuropathy, Immobility syndrome

\section{Background}

The hospitalization in an Intensive Care Unit may lead to important structural and functional deficits, resulting in high morbidity and mortality. This functional decline is largely due to immobility. Until 1950, absolute rest was part of the treatment of almost all diseases. With the progressive evidence of the several undesirable effects in organs and systems observed with prolonged rest, this premise was replaced by the greater encouragement of mobilization, which is increasingly advocated to start earlier, since clinical stability [1].

\section{Methods}

Narrative review of the literature published until February 2018 in the Medline, Embase, Cochrane Library, Web of Science and Scopus databases.

For the search, we considered the following key words: Immobility syndrome, amyotrophy, myopathy, neuropathy, intensive care, critically ill patients, mobilization, exercise, rehabilitation and physiotherapy. We included all articles concerning the effect of the prolonged rest and its impact in muscle and nerve functions, as well as the benefits and safety of the early mobilization in the context of critically ill patients.

\section{Results}

Studies have shown that amyotrophy (a progressive wasting of muscle tissues) can be installed soon after some hours of immobility. The prolonged rest in a bed for only one week is sufficient to decrease muscle mass by $30 \%$ [2]. Amyotrophy, muscle strength deficit, sarcopenia (a condition characterized by loss of skeletal muscle mass and function), pain, contractures and osteoporosis are some of the musculoskeletal complications frequently observed in critically ill patients [3]. However, the negative effects of immobility are rarely limited to a single system. Numerous other comorbidities may result from hospitalization in an Intensive Care Unit: Cardiovascular (hypotension, corporal fluid redistribu-

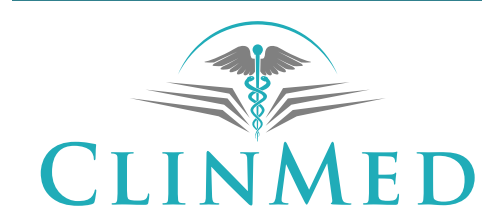

INTERNATIONAL LIBRARY
Citation: Zão A (2018) The Role of Early Mobilization in the Intensive Care. Int J Physiatry 4:014. doi. org/10.23937/2572-4215.1510014

Accepted: June 05, 2018: Published: June 07, 2018

Copyright: (C) 2018 Zão A, et al. This is an open-access article distributed under the terms of the Creative Commons Attribution License, which permits unrestricted use, distribution, and reproduction in any medium, provided the original author and source are credited. 
tion, cadiac function changes and thrombotic events), respiratory (changes in the ratio ventilation/perfusion, restrictive syndrome, respiratory secretions accumulation, atelectasis, respiratory infection), genito-urinary (lower urinary tract dysfunction, urinary infection, renal lithiasis), gastrointestinal (desnutrition, gastroesophasic reflux, constipation), endocrine and metabolic (hyperglycemia, lipidic profile changes, hypercatabolic state, hypoproteinemia and hypoalbuminemia), immune, cutaneous (pressure ulcers), neurological and cognitive-behavioral (sleep disorders, depression, irritability, anxiety, memory changes, lack of concentration and attention and decrease of muscle strength, neuromotor coordination and balance) [4-8].

Immobility syndrome, myopathy, and neuropathy are serious complications commonly found in ICUs. Approximately $15-35 \%$ of patients admitted to an Intensive Care Unit will develop myopathy and about 50\% to $70 \%$ of patients with sepsis, multiorganic dysfunction or prolonged mechanical ventilation will have neuromuscular dysfunction [9]. Critical illness patient mechanically ventilated for more than 7 days have increased mortality risk (48-84\% versus $19-56 \%$ comparing to no mechanically ventilated patients) [10]. Intensive care-associated myopathy is an acute myopathy defined by the loss of myosin filaments and atrophy of muscle fibers, especially type II, resulting in a more evident deficit of muscle strength at the proximal level [2]. On the other hand, neuropathy is caused by axonal degeneration, both of motor and sensory nerve fibers, resulting in muscle strength deficit, especially distally. The pathogenesis of polyneuromyopathy is complex and envolves microvascular, metabolic and electrical changes and bioenergetic failure. Thus, the etiology is multifactorial and several risk factors have been described, such as: The severity and duration of the systemic inflammatory response syndrome, high score in the Acute Physiologic Assessment and Chronic Health Evaluation, sepsis, multiorgan dysfunction, hyperglycemia, hypoalbuminemia, immobility, parenteral nutrition, corticoids, duration of the hospitalization, female gender and age [11]. Diagnosis is based in clinical presentation and may be confirmed by electrophysiology studies [10]. It is fundamental the clinical suspection to other differential diagnosis, such as: Stroke, epidural abscess, Guillain Barre Syndrome, other neuropathies, rabdomiolisis, pharmacological iatrogenia (neurotoxicity, cortisol myopathy) $[7,8,10]$.

The evidence about the benefits of early mobilization and exercise in critically ill patients is growing in the literature, focusing on different systems [12]. Concerning osteoarticular and musculoskeletal field, the benefits refer to the maintenance and gain of joint range of motion, increase of muscular trophism, maintenance of muscular length, maintenance and gain of muscle strength, decrease of contractures and pain $[12,13]$. Regarding neurological setting, exercise and mobilization seems to improve disorders of consciousness and com- portamental, emotional and cognitive functions (namely attention and executive functions), improve muscular strength, neuromotor coordination, balance and gait. In the cardiovascular field, literature highlight the optimization of the autonomic system and the increase of the venous return and the cardiac output, as well as, the reconditioning to the effort. Respiratory benefits refer to the improvement of oxygen transport, increase of tidal volume, increase in thoracic expansion and improvement in respiratory secretion drainage [13-16].

All of this is orchestrated allowing for functional optimization, reducing the incidence of complications and length of hospitalization, leading to a higher quality of life and reducing the health care associated costs. Recent studies also shown that early mobilization is not only beneficial, but also very safe, even in the initial stages of Intensive Care Unit stay, provided that the patient meets the minimum requirements for physiological, cardiorespiratory and neurological stability already well defined in the literature $[1,3,12,13]$.

\section{Conclusion}

Immobility syndrome, myopathy and neuropathy are potentially serious complications commonly found in ICUs, which delay the recovery of the underlying disease itself and can lead to significant functional limitations, restriction of activities, reduction of patients' participation and integration in their social, professional and family contexts. The recognition of risk factors and early diagnosis and intervention are imperative for the successful recovery of these patients.

\section{Declarations}

\section{Ethics approval and consent to participate}

Not applicable.

\section{Competing interests}

The author declares that she has no competing interests.

\section{Funding}

The author declares there is no funding.

\section{References}

1. Cameron S, Ball I, Cepinskas G, Karen Choong, Timothy J Doherty, et al. (2015) Early mobilization in the critical care unit: A review of adult and pediatric literature. Journal Crit Care 30: 664-672.

2. Zhou C, Limin W, Fengming N, Wei Ji, Jiang Wu, et al. (2014) Critical illness polyneuropathy and myopathy: A systematic review. Neural Regen Res 9: 101-110.

3. Hermans G, Van den Berghe G (2015) Clinical review: Intensive care unit acquired weakness. Crit Care 19: 274.

4. Koshy K, Zochodne D (2013) Neuromuscular complications of critical illness. Handb Clin Neurol 115: 759-780.

5. Argov Z, Latronico N (2014) Neuromuscular complications in intensive care patients. Handb Clin Neurol 121: 1673-1685. 
6. Shepherd S, Batra A, Lerner D (2017) Review of critical illness myopathy and neuropathy. Neurohospitalist 7: 41-48.

7. Dos Santos C, Batt J (2012) ICU-acquired weakness: Mechanisms of disability. Curr Opin Crit Care 18: 509-517.

8. Friedrich O, Reid M, Van den Berghe G, Vanhorebeek I, Hermans G, et al. (2015) The sick and the weak: Neuropathies/ Myopathies in the critically ill. Physiol Rev 95: 1025-1109.

9. Rao M, Marella P, Kath B (2008) Assessment of severity and outcome of critical illness. Indian J Anesthesia 52: 652-662.

10. Parker A, Sricharoenchai T, Needham D (2013) Early rehabilitation in the intensive care unit: Preventing physical and mental health impairments. Curr Phys Med Rehabil Reports 1: 307-314.

11. Mehrholz J, Pohl M, Kugler J, Burridge J, Mückel S, et al. (2015) Physical rehabilitation for critical illness myo- pathy and neuropathy. Cochrane Database Syst Rev CD010942.

12. Zorowitz RD (2016) ICU-Acquired weakness: A rehabilitation perspective of diagnosis, treatment, and functional management. Chest 150: 966-971.

13. Latronico N, Guarneri B (2008) Critical illness myopathy and neuropathy. Minerva Anestesiol 74: 319-323.

14. Taito S, Shime N, Ota K, Hideto Yasuda (2016) Early mobilization of mechanically ventilated patients in the intensive care unit. J Intensive Care 4: 50.

15. Semmler A, Okulla T, Kaiser M, Seifert B, Heneka MT (2013) Long-term neuromuscular sequelae of critical illness. J Neurol 260: 151-157.

16. Adler J, Malone D (2012) Early mobilization in the intensive care unit: A systematic review. Cardiopulm Phys Ther J 23: $5-13$. 